EGU2020-3476, updated on 10 Mar 2020

https://doi.org/10.5194/egusphere-egu2020-3476

EGU General Assembly 2020

(c) Author(s) 2020. This work is distributed under

the Creative Commons Attribution 4.0 License.

\title{
Diverging impacts of extreme summers on European C-cycling from different regional and seasonal compensation effects
}

\author{
Ana Bastos ${ }^{1}$ and the ICOS drought task-force* \\ ${ }^{1}$ Ludwig Maximilians University, Geography Dept., München, Germany (ana.bastos@Imu.de)
}

${ }^{*}$ A full list of authors appears at the end of the abstract

In the past 20 years, three major drought-heat events (DH) occurred in Europe: DH2003 in western Europe, DH2010 in western Russia and, more recently, DH2018 in central Europe and Scandinavia. These events were all preceded by warm and dry springs that contributed to the summer heatwaves and are comparable in magnitude. However, they varied in their geographical distribution and biomes affected, so they can be used as "natural experiments" to improve our understanding of ecosystems' responses to climate conditions that will become more frequent in the coming decades.

We analyze anomalies in carbon, water and energy fluxes from 11 Dynamic Global Vegetation Models (DGVMs) forced with higher spatial and temporal resolution climate forcing than conventional global simulations, as well as an ensemble of estimates from the data driven FLUXCOM product. All three DH events were associated with decreases in summer gross primary productivity (GPP), but the relative strength at continental scale differed between events. The DGVMs and FLUXCOM show event-dependent agreement in estimated gross and net $\mathrm{CO}_{2}$ fluxes. We also find a progressively stronger negative effect of heat anomalies on ecosystem productivity between each event, which might indicate a transition towards more water-limited regime at continental scale by progressively warmer background conditions.

The different impacts of the three DH events on continental-scale summer GPP are in part related to regional asymmetries in climate anomalies that act to amplify or offset the impact of DH events on ecosystem productivity, depending on their geographical distribution and biomes affected. At the annual scale, both FLUXCOM and DGVMs indicate close to neutral or above-average $\mathrm{CO}_{2}$ uptake in the three years, when removing the long-term trend. This is in part because increased productivity in spring in response to warming and reduced respiration in autumn compensated for less photosynthetic uptake in summer.

All models show good skill in simulating the soil-moisture anomalies during DH events, but in general DGVMs show poor skill in simulating the increased sensitivity of GPP to soil-moisture and decreased sensitivity to temperature during extreme events. The relative skill of individual DGVMs in simulating these changes of GPP sensitivity to climate during extreme events explains the mismatch in simulated productivity anomalies with FLUXCOM estimates. 
ICOS drought task-force: Z. Fu 2, P. Ciais 2, S. Sitch 3, P. Friedlingstein 4, J. Pongratz 1, Ulrich Weber 5, Markus Reichstein 5, P. Anthoni 6, A. Arneth 6, V. Haverd 7, A. Jain 8, E. Joetzjer 9, Jürgen Knauer 7, S. Lienert 10, T. Loughran 1, Ryan Padrón Flasher 11, P.C. McGuire 12, W. Obermeier 1, H. Tian 13, N. Viovy 2, Sönke Zaehle 5, Lei Fan 14, Jean-Pierre Wigneron 14 